\title{
Evaluation of the European Committee on Antimicrobial Susceptibility Testing Guidelines for Rapid Antimicrobial Susceptibility Testing of Bacillus anthracis-, Yersinia pestis- and Francisella tularensis-Positive Blood Cultures
}

\author{
Ohad Shifman ${ }^{1, *,+} \mathbb{D}$, Tamar Aminov ${ }^{1,+}{ }^{,}$Moshe Aftalion ${ }^{1}$, David Gur ${ }^{1}$, Hila Cohen ${ }^{1}$, Elad Bar-David ${ }^{2}$, \\ Ofer Cohen ${ }^{1}$, Emanuelle Mamroud ${ }^{1}$ (D), Haim Levy ${ }^{2}{ }^{(D)}$, Ronit Aloni-Grinstein ${ }^{1}$ (D) Ida Steinberger-Levy ${ }^{1}(\mathbb{D})$ \\ and Shahar Rotem ${ }^{1, *}$
}

1 The Department of Biochemistry and Molecular Genetics, Israel Institute for Biological Research, Ness Ziona 7410001, Israel; tamara@iibr.gov.il (T.A.); moshea@iibr.gov.il (M.A.); gurd@iibr.gov.il (D.G.); hilac@iibr.gov.il (H.C.); oferc@iibr.gov.il (O.C.); emmym@iibr.gov.il (E.M.); ronitag@iibr.gov.il (R.A.-G.); idasl@iibr.gov.il (I.S.-L.)

2 The Department of Infectious Diseases, Israel Institute for Biological Research, Ness Ziona 7410001, Israel; eladb@iibr.gov.il (E.B.-D.); haiml@iibr.gov.il (H.L.)

check for

updates

Citation: Shifman, O.; Aminov, T.; Aftalion, M.; Gur, D.; Cohen, H.; Bar-David, E.; Cohen, O.; Mamroud, E.; Levy, H.; Aloni-Grinstein, R.; et al. Evaluation of the European Committee on Antimicrobial Susceptibility Testing Guidelines for Rapid Antimicrobial Susceptibility Testing of Bacillus anthracis-, Yersinia pestis- and Francisella tularensis-Positive Blood Cultures. Microorganisms 2021, 9, 1055. https://doi.org/10.3390/ microorganisms 9051055

Academic Editor: Suresh G. Joshi

Received: 19 April 2021

Accepted: 10 May 2021

Published: 13 May 2021

Publisher's Note: MDPI stays neutral with regard to jurisdictional claims in published maps and institutional affiliations.

Copyright: (C) 2021 by the authors. Licensee MDPI, Basel, Switzerland. This article is an open access article distributed under the terms and conditions of the Creative Commons Attribution (CC BY) license (https:/ / creativecommons.org/licenses/by/ $4.0 /)$.
* Correspondence: ohads@iibr.gov.il (O.S.); shaharr@iibr.gov.il (S.R.)

$\dagger$ Contributed equally to this study.

Abstract: Rapid determination of bacterial antibiotic susceptibility is important for proper treatment of infections. The European Committee on Antimicrobial Susceptibility Testing (EUCAST) has recently published guidelines for rapid antimicrobial susceptibility testing (RAST) performed directly from positive blood culture vials. These guidelines, however, were only published for a limited number of common pathogenic bacteria. In this study, we evaluated the applicability of these guidelines to three Tier 1 bioterror agents (Bacillus anthracis, Yersinia pestis and Francisella tularensis) that require prompt antibiotic treatment to mitigate morbidity and mortality. We used spiked-in human blood incubated in a BACTEC ${ }^{\mathrm{TM}}$ FX40 system to determine the proper conditions for RAST using disc-diffusion and Etest assays. We found that reliable disc-diffusion inhibition diameters and Etest MIC values could be obtained in remarkably short times. Compared to the EUCASTrecommended disc-diffusion assays that will require adjusted clinical breakpoint tables, Etest-based RAST was advantageous, as the obtained MIC values were similar to the standard MIC values, enabling the use of established category breakpoint tables. Our results demonstrate the promising applicability of the EUCAST RAST for B. anthracis-, Y. pestis- or F. tularensis-positive blood cultures, which can lead to shorter diagnostics and prompt antibiotic treatment of these dangerous pathogens.

Keywords: blood culture; Bacillus anthracis; Yersinia pestis; Francisella tularensis; rapid antimicrobial susceptibility testing; RAST; EUCAST; Etest; disc diffusion; AST

\section{Introduction}

B. anthracis, $Y$. pestis and F. tularensis are three pathogens that are categorized by the U.S. Centers for Disease Control and Prevention (CDC) as Tier 1 biological select agents [1]. Without proper treatment, high rates of mortality are observed within a short period following infection [2-5]. These characteristics led to the recognition of these pathogens as potential biological threat agents. Anthrax, the disease caused by B. anthracis, can appear in three major forms: cutaneous, gastrointestinal and inhalational. Although a vaccine was developed against $B$. anthracis, vaccination is not routinely given, and antibiotic therapy is the treatment of choice following exposure. Without treatment, anthrax progresses rapidly and can be fatal in most cases [4-6]. Y. pestis is the etiological agent of plague, a severe and fatal disease. This disease has several major forms, bubonic, pneumonic and septicemic, 
depending on the route of exposure [2,5]. In the absence of proper antibiotic treatment administered within 18 to $24 \mathrm{~h}$ of symptom onset, high rates of mortality are observed. F. tularensis is the etiological cause of tularemia. Tularemia can also appear in several major forms of illness: ulceroglandular, glandular, oculoglandular, oropharyngeal, typhoid and pneumonic. Without proper antibiotic treatment, tularemia can result in mortality rates of up to $60 \%$, and antibiotic therapy can reduce these rates to $1 \%$ [3,5]. As antibiotic treatment is the major therapeutic tool for these acute and rapidly progressing diseases, there is a concern that antibiotic resistance may result either from natural emergence or from deliberate engineering for a bioterror purpose [7-15]. Thus, there is a necessity for rapid antibiotic susceptibility testing that will offer the correct treatment for these diseases to reduce mortality rates, length of hospital stay and hospitalization costs $[16,17]$.

Blood cultures (BCs) are the main source for bacterial isolation for antimicrobial susceptibility testing (AST) purposes. Conventional AST from positive BCs requires a concentration-defined suspension of bacteria, which is achieved by plating and incubating a sample of the blood culture on agar plates [18]. Incubation on agar plates also reduces possible growth inhibition that may be caused by blood components, antibiotics administered to the patient or BC media supplementations, which may affect the AST results. However, the isolation step is time consuming, especially in bacteria that grow slowly in vitro, such as $Y$. pestis and F. tularensis. Over the years, attempts have been made to develop RAST assays [19-22]. However, most of these methods are not standardized, hampering their acceptance in clinical laboratories. Recently, the EUCAST and the Clinical and Laboratory Standards Institute (CLSI) have proposed standard RAST assays based on shortened discdiffusion assays, which are conducted after plating samples obtained directly from BC vials and interpreted by using dedicated susceptibility breakpoint interpretation tables [23-26]. However, these RAST guidelines were validated and published for only a limited number of common life-threatening bacteria that can easily acquire antibiotic resistance (Escherichia coli, Klebsiella pneumoniae, Pseudomonas aeruginosa, Acinetobacter baumannii, Staphylococcus aureus, Enterococcus faecalis, Enterococcus faecium and Streptococcus pneumoniae) [26]. Other studies have shown the feasibility of these approaches for additional bacteria, but also highlighted the importance of determining whether this direct method is implementable for each bacterial-antibiotic combination [23,25,27-34].

In this study, we examined the applicability of the RAST approach to B. anthracis, Y. pestis and F. tularensis, three Tier 1 select biological agents, which have not been evaluated before. As bacteremia is evident for all three diseases caused by these bacteria, establishing a standard RAST directly from BCs can accelerate proper treatment and recovery. We used human blood cultures spiked with either B. anthracis, Y. pestis or F. tularensis. We then plated samples taken directly from the BCs for determination of the optimal conditions for proper RAST using disc-diffusion and Etest assays. We found that by using Etest-based RAST, reliable MIC results can be obtained for the recommended therapeutic antibiotics, following 6 to $8 \mathrm{~h}, 18$ to $24 \mathrm{~h}$ and 24 to $30 \mathrm{~h}$ of incubation for B. anthracis, Y. pestis and F. tularensis, respectively. These results suggest that RAST can be successfully applied for these Tier 1 select agents.

\section{Materials and Methods}

\subsection{Bacterial Strains, Growth Conditions and Colony Forming Unit Determination}

B. anthracis Vollum $\mathrm{PXO1}^{-} \mathrm{pXO2}{ }^{-}$spores [35] were germinated to vegetative form in terrific broth [36] at $37^{\circ} \mathrm{C}$ for $30 \mathrm{~min}$ before use. The Y. pestis EV76 vaccine strain [37] was routinely grown on brain heart infusion agar (BHIA, BD Cat \#241830) plates at $28^{\circ} \mathrm{C}$. The F. tularensis live vaccine strain (LVS, ATCC 29684) was grown on Cystine Heart Agar (CHA) plates (BD Cat \#247100) enriched with $1 \%(w / v)$ hemoglobin (BD, Cat \#212392) at $37^{\circ} \mathrm{C}$. Colony forming units (CFUs) were counted by drop plating, in triplicate, $10 \mu \mathrm{L}$ from serial tenfold culture dilutions in sterile Dulbecco's phosphate-buffered saline (PBS, Biological Industries Cat \#02-023-1A) on Muller Hinton agar (MHA, BD Cat \#225250), BHIA and CHA plates for B. anthracis, Y. pestis and F. tularensis, respectively. 


\subsection{Antibiotics}

Antibiotics for disc-diffusion AST assays were purchased from BD and Oxoid; Etest strips were purchased from bioMerieux, Marcy l'Etoile, France. The antibiotic concentrations, catalog numbers and abbreviations used in this study are given in Table 1 . The antibiotics were stored according to the manufacturers' instructions until use.

Table 1. Antibiotics used in this study.

\begin{tabular}{|c|c|c|c|c|}
\hline Antibiotics & $\begin{array}{c}\text { Etest } \\
\text { Concentration Range } \\
(\mu \mathrm{g} / \mathrm{mL})\end{array}$ & Cat \# & $\begin{array}{c}\text { Disc } \\
\text { Concentration }(\mu \mathrm{g})\end{array}$ & Cat \# \\
\hline Ciprofloxacin (CIP) & $0.002-32$ & 412311 & 5 & $231657^{a}$ \\
\hline Doxycycline (DOX) & $0.016-256$ & 412328 & - & - \\
\hline Tetracycline (TET) & - & - & 30 & $230998^{a}$ \\
\hline Chloramphenicol (CHL) & $0.016-256$ & 412309 & 30 & СТ0013B ${ }^{b}$ \\
\hline Gentamicin (GEN) & $0.016-256$ & 412368 & 10 & $231227^{a}$ \\
\hline Streptomycin (STR) & $0.064-1024$ & 526800 & - & - \\
\hline Trimethoprim-Sulfamethoxazole (TRS) & $0.002-32$ & 412481 & $1.25 / 23.75$ & СТ0052B ${ }^{b}$ \\
\hline Amoxicillin-Clavulanic acid (AMC) & - & - & $20 / 10$ & $231628^{a}$ \\
\hline Ampicillin (AMP) & $0.016-256$ & 412253 & - & - \\
\hline Clarithromycin (CLA) & $0.016-256$ & 412313 & - & - \\
\hline Clindamycin (CLI) & $0.016-256$ & 412315 & - & - \\
\hline Imipenem (IMI) & $0.002-32$ & 412374 & 10 & $231644^{\mathrm{a}}$ \\
\hline Levofloxacin (LEV) & - & - & 5 & $231705^{a}$ \\
\hline Linezolid (LIN) & - & - & 30 & $231761^{a}$ \\
\hline Moxifloxacin (MOX) & - & - & 5 & $231757^{a}$ \\
\hline Penicillin G (BEN) & - & - & 1 Unit & СТ0152B ${ }^{b}$ \\
\hline Rifampicin (RIF) & $0.002-32$ & 412450 & - & - \\
\hline Vancomycin (VAN) & $0.016-256$ & 412488 & - & - \\
\hline
\end{tabular}

${ }^{\mathrm{a}} \mathrm{BD} ;{ }^{\mathrm{b}}$ Oxoid.

\subsection{Antimicrobial Susceptibility Testing}

Standard AST was performed by spreading $125 \mu \mathrm{L}$ of bacterial suspension $\left(\sim 10^{8} \mathrm{CFU} / \mathrm{mL}\right)$ on $90 \mathrm{~mm}$ agar plates (MHA for B. anthracis and Y. pestis, CHA for F. tularensis) using a Drigalski spatula and application of 2-3 discs or 1-2 Etest strips on each of the swabbed plates. The plates were incubated at $35^{\circ} \mathrm{C}$, and standard values were determined at 18 and $20 \mathrm{~h}$ (B. anthracis) or $24 \mathrm{~h}$ (Y. pestis). F. tularensis-seeded plates were incubated at $35^{\circ} \mathrm{C}$ with $5 \% \mathrm{CO}_{2}$ for $48 \mathrm{~h}$. RAST was performed by spreading $125 \mu \mathrm{L}$ of culture sampled from a positive BC vial on $90 \mathrm{~mm}$ agar plates using a Drigalski spatula and applying antibiotic discs or Etest strips on the swabbed plates. The plates were incubated under the same conditions as used for the standard AST.

\subsection{MIC and Inhibition Zone Diameter Determination}

For the Etest assays, the MIC values were determined by reading the scale values on the strips at the intersection of the growth inhibition zone (from the front of the plate with the lid removed) according to the manufacturer's guidelines. MIC values were rounded up to the nearest twofold antibiotic concentration. For the disc-diffusion tests, the inhibition zone diameters were measured from the front of the plate with the lid removed according to EUCAST guidelines.

\section{Results}

Recently, EUCAST published guidelines for the rapid determination of antibiotic susceptibility directly from BCs. The improvement in the assay duration was achieved by addressing two aspects. First, bacteria were plated on agar plates directly from the BC vials without prior isolation, enrichment and quantification of the bacterial inoculum, steps that are time consuming and labor intensive. Second, the antibiotic inhibition zones were 
read at earlier times ( 4 to $8 \mathrm{~h}$ ) compared to the standard reading times (18 to $20 \mathrm{~h}$ ). We sought to evaluate whether these shortcuts could also be applied to B. anthracis, Y. pestis and F. tularensis, three Tier 1 select agents on the CDC list for which RAST can be beneficial. We first analyzed the applicability for B. anthracis. A similar analysis for $Y$. pestis and F. tularensis will be presented thereafter.

\subsection{Bacillus anthracis}

\subsubsection{Early Reading of AST Results}

First, we wanted to determine the earliest time points for which reliable AST results can be obtained for $B$. anthracis. The reading times are affected by the ability to visually examine, by an unaided eye, the growth of the bacteria and, hence, the determination of inhibition zones and the change in the inhibition zone over time. To this end, we conducted disc-diffusion assays with a panel of antibiotics, including the CLSIand EUCAST-recommended antibiotics (CIP, TET and BEN) and five additional potential therapeutic antibiotics (Table 2). Using a suspension of germinated B. anthracis spores at the standard inoculum concentration of $1 \times 10^{8} \mathrm{CFU} / \mathrm{mL}$, we determined the inhibition zone diameters at various time points, including early reading time points, as suggested by the EUCAST RAST guidelines ( 6 to $8 \mathrm{~h}$ ) and the standard reading time points (18 to $20 \mathrm{~h}$ ). Distinct inhibition edges were evident as early as $6 \mathrm{~h}$ of incubation for all the tested antibiotics. However, for most of the tested antibiotics, the inhibition zone diameters differed between the shortened reading times ( 6 to $8 \mathrm{~h}$ ) and the recommended reading times for standard tests (18 to $20 \mathrm{~h}$ ). These results suggest that reading results at shortened time points is feasible, but specific susceptibility breakpoint criteria should be determined for the shortened incubation for RAST implementation. We also tested the possibility of determining MIC values using Etest strips for additional antibiotic substances (Table 3). For the disc-diffusion-based assay, the edges of the inhibition zone could be visible after $6 \mathrm{~h}$ of incubation. In these assays, however, the MIC values for most antibiotics (CIP, AMP, CHL, CLI and CLA) at early reading times (6 to $8 \mathrm{~h}$ ) and standard reading times (18 to $20 \mathrm{~h}$ ) were similar; thus, susceptibility category interpretation for RAST can be based on the standard breakpoint interpretation tables [38] or standard AST results. DOX, VAN and RIF were the only antibiotics in which their MIC values at shortened reading times were different from those obtained at the standard times; thus, even Etest-based RAST implementation will require dedicated breakpoint interpretation tables.

Table 2. Disc-diffusion inhibition zones of germinated B. anthracis spores at various time points.

\begin{tabular}{ccccccccc}
\hline \multirow{2}{*}{$\begin{array}{c}\text { Incubation } \\
\text { Duration (h) }\end{array}$} & \multicolumn{7}{c}{ Inhibition Zone Diameter (mm) } \\
\cline { 2 - 9 } & CIP & TET & BEN & AMC & LIN & MOX & LEV & IMI \\
\hline 6 & 21 & $25-26$ & $19-20$ & 28 & 21 & 17 & 17 & 31 \\
8 & $22-25$ & $27-28$ & 20 & 30 & 30 & 17 & 23 & 34 \\
16 & 26 & 30 & 21 & 31 & 28 & 23 & 25 & 38 \\
18 & 26 & 30 & 21 & 32 & 28 & 23 & 26 & 39 \\
20 & 26 & 30 & 21 & 33 & 28 & 23 & 26 & 39 \\
23 & $24-26$ & $29-30$ & $20-21$ & $30-33$ & $28-30$ & 23 & 26 & $39-40$ \\
\hline
\end{tabular}

CIP-ciprofloxacin; TET-tetracycline; BEN-penicillin G; AMC-amoxicillin-clavulanate; LIN-linezolid; MOX_-moxifloxacin; LEV_levofloxacin; IMI-imipenem; the inhibition zone diameters were determined by at least three independent assays.

\subsubsection{Characterization of B. anthracis Growth in Human Blood Culture Vials}

Before applying RAST to B. anthracis-grown BCs, we characterized the correlation between the inoculum concentration and the incubation time needed for the BCs to be flagged as positive. In addition, we determined the bacterial concentrations when BCs flagged positive. We spiked human blood with $B$. anthracis at a wide range of concentrations $\left(1 \times 10^{1}\right.$ to $\left.1 \times 10^{5} \mathrm{CFU} / \mathrm{mL}\right)$ and transferred the spiked blood into BACTEC ${ }^{\mathrm{TM}}$ Plus Aerobic/F culture vials. The samples were then incubated in a BACTEC ${ }^{\text {TM }}$ FX40 device 
until they were flagged as positive by the device. At this point, the bacterial concentrations were determined by CFU counting (Table 4).

Table 3. Etest MIC values of germinated B. anthracis spores at various time points.

\begin{tabular}{ccccccccc}
\hline \multirow{2}{*}{$\begin{array}{c}\text { Incubation Duration } \\
\text { (h) }\end{array}$} & CIP & DOX & AMP & CHL & CLI & CLA & VAN & RIF \\
\cline { 2 - 9 } & 0.06 & $<0.016$ & $0.03-0.06$ & $4-8$ & $0.125-0.5$ & $0.25-0.5$ & 0.125 & $0.03-0.06$ \\
6 & 0.06 & $\leq 0.016$ & $0.03-0.06$ & $4-8$ & $0.125-0.5$ & $0.25-0.5$ & 0.25 & $0.03-0.125$ \\
8 & 0.06 & $\leq 0.016$ & 0.06 & $4-8$ & 0.25 & 0.25 & 0.25 & 0.06 \\
10 & 0.06 & $0.016-0.06$ & $0.06-0.125$ & 4 & $0.25-0.5$ & 0.25 & 1 & $0.25-0.5$ \\
16 & 0.06 & $0.016-0.125$ & $0.06-0.125$ & $4-8$ & $0.25-0.5$ & 0.25 & $1-2$ & $0.25-0.5$ \\
18 & 0.06 & $0.06-0.125$ & $0.06-0.125$ & $4-8$ & $0.25-0.5$ & 0.25 & $1-2$ & 0.5 \\
20 & 0.06 & $0.06-0.125$ & $0.06-0.125$ & 8 & $0.25-0.5$ & $0.25-0.5$ & $1-2$ & $0.25-0.5$ \\
23 & & &
\end{tabular}

CIP-ciprofloxacin; DOX—doxycycline; AMP—ampicillin; CHL—chloramphenicol; CLI—clindamycin; CLA—clarithromycin; VAN— vancomycin; RIF-rifampicin; the MIC values were determined by at least three independent assays.

Table 4. Characterization of B. anthracis growth in blood culture vials.

\begin{tabular}{|c|c|c|c|c|}
\hline $\begin{array}{l}\text { Initial Concentration } \\
\text { in Blood (CFU/mL) }\end{array}$ & $\begin{array}{l}\text { Incubation Duration } \\
\text { until the Alert } \\
\text { (hh: } \mathrm{mm})\end{array}$ & $\begin{array}{l}\text { Concentration at the } \\
\text { Alert }(\mathrm{CFU} / \mathrm{mL})\end{array}$ & $\begin{array}{l}\text { Concentration, } 18 \mathrm{~h} \\
\text { after the Alert, } \\
\text { Incubation in the } \\
\text { Device (CFU/mL) }\end{array}$ & $\begin{array}{c}\text { Concentration, } 3 \mathrm{~h} \text { after the } \\
\text { Alert, at Room } \\
\text { Temperature (CFU/mL) }\end{array}$ \\
\hline $1.0 \times 10^{5}$ & $4: 30$ to $6: 30$ & $8.7 \times 10^{6}$ to $2.9 \times 10^{7}$ & \multirow{5}{*}{$2.0 \times 10^{7}$ to $7.0 \times 10^{7}$} & \multirow{5}{*}{$2.3 \times 10^{7}$ to $5.0 \times 10^{7}$} \\
\hline $1.0 \times 10^{4}$ & $6: 30$ to $7: 50$ & $8.0 \times 10^{6}$ to $1.5 \times 10^{7}$ & & \\
\hline $1.0 \times 10^{3}$ & $7: 50$ to $9: 40$ & \multirow{2}{*}{$6.0 \times 10^{6}$ to $2.4 \times 10^{7}$} & & \\
\hline $1.0 \times 10^{2}$ & $9: 40$ to $10: 30$ & & & \\
\hline $1.0 \times 10^{1}$ & $10: 50$ to $11: 50$ & $3.8 \times 10^{6}$ to $1.8 \times 10^{7}$ & & \\
\hline
\end{tabular}

The values were determined by at least three independent assays.

Remarkably, despite the large difference in the initial B. anthracis concentrations, the bacterial concentrations at the alert were relatively similar and in a narrow range of $6.0 \times 10^{6}$ to $2.9 \times 10^{7} \mathrm{CFU} / \mathrm{mL}$. This was a consequence of prolongation in the incubation time needed for the culture to turn positive at lower inoculum concentrations, with a range of 4.5 to $6.5 \mathrm{~h}$ needed for the highest-spiked inoculum of $1.0 \times 10^{5} \mathrm{CFU} / \mathrm{mL}$ to 11 to $12 \mathrm{~h}$ for the lowest spiked inoculum of $1.0 \times 10^{1} \mathrm{CFU} / \mathrm{mL}$. We also examined the bacterial concentrations at the maximal postponement after the alert allowed by the EUCAST regulations before applying RAST (18 $\mathrm{h}$ of incubation in the device or $3 \mathrm{~h}$ at room temperature). In both cases, bacterial viability was maintained, as bacterial CFUs were at least as high as those at the alert, suggesting that RAST can be evaluated for these time frames.

\subsubsection{RAST Directly from Positive Blood Culture Vials}

Finally, we wanted to evaluate the EUCAST method for RAST directly from B. anthracis-positive BC vials. According to the EUCAST guidelines, RAST can be performed 0 to $18 \mathrm{~h}$ after BCs have signaled positive, provided that during this period, the BC vials were not removed from the device. In cases where the vials are removed from the device and kept at room temperature, RAST can be performed up to $3 \mathrm{~h}$ thereafter. Accordingly, we examined the RAST performance at three time points: when the vials were flagged as positive, $18 \mathrm{~h}$ after they were positively flagged and were left in the device, and $3 \mathrm{~h}$ after they were positively flagged and were kept at room temperature. The antibiotics used were those analyzed before using the standard inoculum culture. For all the antibiotics tested, including ciprofloxacin (Table 5), tetracycline, penicillin G, amoxicillin-clavulanic acid, linezolid, moxifloxacin, levofloxacin and imipenem (Supplementary Tables S1-S7), the inhibition zone diameters obtained by RAST directly from positive BCs ("at the alert" columns) 
were similar to the diameters obtained for the standard culture at the corresponding reading times ("standard culture" columns).

Table 5. Ciprofloxacin inhibition zone diameters of B. anthracis-positive blood cultures.

\begin{tabular}{ccccc}
\hline & \multicolumn{4}{c}{ Inhibition Zone Diameter (mm) } \\
\hline Incubation Duration (h) & Standard Culture ${ }^{\text {a }}$ & At the Alert & $\begin{array}{c}\text { 18 h after the Alert, } \\
\text { Incubation in the Device }\end{array}$ & $\begin{array}{c}\text { 3 h after the Alert, } \\
\text { at Room Temperature }\end{array}$ \\
\hline 6 & 21 & $20-21$ & $20-21$ & $18-21$ \\
8 & $22-25$ & $23-25$ & $22-24$ & $20-22$ \\
16 & 26 & $23-26$ & ND & ND \\
18 & 26 & $23-26$ & ND & ND \\
20 & 26 & $23-26$ & $22-25$ & $21-24$ \\
23 & $24-26$ & $23-26$ & \\
\hline
\end{tabular}

${ }^{\text {a }}$ Ciprofloxacin inhibition-zone diameter for the standard culture were taken from Table 2; ND—not determined; the diameters were determined by at least three independent assays.

As was observed for the standard culture, for most antibiotics, the diameters at the shortened reading times were different from those of the longer reading times. These results suggest that although RAST performed directly from BCs is feasible for B. anthracis, specific interpretation breakpoint tables should be established for shorter reading times. Postponement of the culture, either $18 \mathrm{~h}$ in the device or $3 \mathrm{~h}$ at room temperature, did not affect the inhibition zone diameters (Table 5, " $18 \mathrm{~h}$ after the alert" and " $3 \mathrm{~h}$ after the alert" columns). Thus, RAST can be performed according to the EUCAST time windows.

We also performed Etest assays directly from these BCs and compared the MIC values to those that we acquired previously using the standard inoculum (Section 3.1.1). For all antibiotics tested-ciprofloxacin (Table 6), doxycycline, ampicillin, clarithromycin, chloramphenicol, clindamycin, vancomycin and rifampicin (Supplementary Tables S8-S14)—there was good agreement between the MIC values obtained from BCs and the MIC values that were obtained by the standard AST. These results also hold true for vials kept $18 \mathrm{~h}$ in the device or $3 \mathrm{~h}$ at room temperature, the maximal postponement length windows allowed by EUCAST regulations. Therefore, our results suggest that Etest-based RAST can be applied directly from $B$. anthracis-positive $\mathrm{BCs}$, starting from $6 \mathrm{~h}$ following plating.

Table 6. Etest ciprofloxacin MIC values for B. anthracis-positive blood cultures.

\begin{tabular}{ccccc}
\hline & \multicolumn{3}{c}{ MIC $(\mu \mathrm{g} / \mathrm{mL})$} \\
$\begin{array}{c}\text { Incubation } \\
\text { Duration (h) }\end{array}$ & Standard Culture ${ }^{\mathbf{a}}$ & At the Alert & $\begin{array}{c}\mathbf{1 8} \mathbf{h} \text { after the Alert, } \\
\text { Incubation in the Device }\end{array}$ & $\begin{array}{c}\mathbf{3} \mathbf{h} \text { after the Alert, } \\
\text { at Room Temperature }\end{array}$ \\
\hline 6 & 0.06 & 0.06 & $0.03-0.06$ & 0.06 \\
8 & 0.06 & 0.06 & $0.03-0.06$ & 0.06 \\
16 & 0.06 & $0.03-0.06$ & ND & ND \\
18 & 0.06 & $0.03-0.06$ & ND & ND \\
20 & 0.06 & $0.03-0.125$ & 0.06 & 0.06 \\
23 & 0.06 & $0.06-0.125$ & ND \\
\hline
\end{tabular}

${ }^{a}$ Ciprofloxacin MIC values for the standard culture were taken from Table 3; ND—not determined; the MIC values were determined by at least three independent assays.

\subsection{Yersinia pestis}

\subsubsection{Early Reading of AST Results}

We first aimed to determine the shortest incubation time for which reliable AST results can be obtained for $Y$. pestis. Therefore, we performed disc-diffusion-based AST using a standard Y. pestis inoculum $\left(\sim 1 \times 10^{8} \mathrm{CFU} / \mathrm{mL}\right.$ originating from isolated colonies $)$ and determined the inhibition zone diameters at different incubation times for a panel of potential therapeutic antibiotics (ciprofloxacin, tetracycline, gentamicin, chloramphenicol 
and trimethoprim-sulfamethoxazole). Distinct inhibition zone edges were evident only after $18 \mathrm{~h}$ of incubation for all tested antibiotics (Table 7).

Table 7. Disc-diffusion inhibition zones of $Y$. pestis at various time points.

\begin{tabular}{cccccc}
\hline Inhibition Zone & \multicolumn{5}{c}{ Inhibition Zone Diameter (mm) } \\
\hline Incubation Duration (h) & CIP & TET & GEN & CHL & TRS \\
\hline 18 & $35-40$ & 28 & $27-28$ & $31-36$ & $40-42$ \\
20 & $36-40$ & $29-30$ & $28-30$ & $30-35$ & $40-42$ \\
22 & $39-41$ & 30 & $28-29$ & $31-36$ & $40-42$ \\
24 & $39-42$ & $30-32$ & $28-30$ & $30-35$ & $39-42$ \\
\hline
\end{tabular}

CIP-ciprofloxacin; TET-tetracycline; GEN-gentamicin; CHL-chloramphenicol; TRS-trimethoprimsulfamethoxazole; the diameters were determined by at least three independent assays.

For CHL and TRS, the inhibition zone diameters were similar between the recommended reading time $(24 \mathrm{~h})$ and the earlier time points, and therefore, the AST can be shortened for these antibiotics. For the other antibiotics (CIP, TET and GEN), the inhibition zone diameters varied slightly between the shortened 18-hour reading time and the standard reading time $(24 \mathrm{~h})$; thus, to shorten the AST, explicit breakpoint tables will be required for the earlier time points. We also checked the possibility of determining MIC values using Etest strips. The Etest MIC values were read at the same time points as for the disc-diffusion assay. Also for the Etest assays, growth inhibition zones were visible only after $18 \mathrm{~h}$ of incubation. However, in contrast to the disc-diffusion test, where only CHL and TRS had similar inhibition zone diameters at the $18 \mathrm{~h}$ and $24 \mathrm{~h}$ readings, Etest assays resulted in similar MIC values for all six tested antibiotics (Table 8). Thus, susceptibility breakpoints could be interpreted following a shortened 18-hour incubation using Y.pestis standard breakpoint tables, such as those published by the CLSI [38].

Table 8. Etest MIC values of Y. pestis at various time points.

\begin{tabular}{ccccccc}
\hline & \multicolumn{7}{c}{ MIC $(\mu \mathrm{g} / \mathrm{mL})$} \\
\hline Incubation Duration (h) & CIP & DOX & GEN & CHL & TRS & STR \\
\hline 18 & 0.016 & $1-2$ & $0.25-1$ & $2-4$ & $0.016-0.03$ & $1-2$ \\
20 & 0.016 & $1-2$ & $0.25-1$ & $2-4$ & $0.016-0.03$ & $1-2$ \\
22 & $0.016-0.03$ & $1-2$ & $0.25-1$ & $2-4$ & $0.016-0.03$ & $1-2$ \\
24 & $0.016-0.03$ & $1-2$ & $0.25-1$ & $2-4$ & $0.016-0.03$ & $1-2$ \\
\hline
\end{tabular}

CIP-ciprofloxacin; DOX-doxycycline; GEN-gentamicin; CHL-chloramphenicol; TRS-trimethoprimsulfamethoxazole; STR—streptomycin; the MIC values were determined by at least three independent assays.

\subsubsection{Characterization of $Y$. pestis Growth in Human Blood Cultures}

Next, we characterized the incubation time needed for $Y$. pestis-spiked BCs to be flagged as positive by the BACTEC ${ }^{\mathrm{TM}}$ FX40 system and determined the bacterial concentrations at that time point. For this purpose, we spiked human blood with $Y$. pestis bacteria at various concentrations $\left(1.4 \times 10^{1}\right.$ to $2.7 \times 10^{3} \mathrm{CFU} / \mathrm{mL}$; Table 9$)$ and transferred the spiked blood into BACTEC ${ }^{\mathrm{TM}}$ Lytic Anaerobic/F culture vials, which were then incubated in a BACTEC ${ }^{\text {TM }}$ FX40 device until they were flagged as positive. BACTEC ${ }^{\text {TM }}$ Lytic Anaerobic/F culture vials were used, as we found that $Y$. pestis grew faster in those vials than in aerobic vials.

As observed for $B$. anthracis, $Y$. pestis concentrations at the alert were similar $\left(1 \times 10^{7}\right.$ to $5.6 \times 10^{7} \mathrm{CFU} / \mathrm{mL}$, "concentration at the alert" column), despite $\sim 2$ orders of magnitude difference in the concentrations of bacteria initially spiked in the vials. This was compensated by a longer incubation duration (from 20-22 h to 25-29 h) for BCs spiked with low bacterial concentrations. We also examined the bacterial concentrations following further incubation after the alert ( $18 \mathrm{~h}$ in the device or $3 \mathrm{~h}$ at room temperature). CFU counting showed that $Y$. pestis, similar to B. anthracis, maintained viability under those conditions, indicating that RAST can be performed in these time frames. 
Table 9. Characterization of Y. pestis growth in blood culture vials.

\begin{tabular}{|c|c|c|c|c|}
\hline $\begin{array}{l}\text { Initial Concentration } \\
\text { in Blood (CFU/mL) }\end{array}$ & $\begin{array}{c}\text { Incubation } \\
\text { Duration until Alert } \\
\text { (hh: } \mathbf{m m})\end{array}$ & $\begin{array}{l}\text { Concentration at the } \\
\text { Alert (CFU/mL) }\end{array}$ & $\begin{array}{c}\text { Concentration, } 18 \mathrm{~h} \\
\text { after the Alert, } \\
\text { Incubation in the } \\
\text { Device (CFU/mL) }\end{array}$ & $\begin{array}{c}\text { Concentration, } 3 \mathrm{~h} \\
\text { after the Alert, at } \\
\text { Room Temperature } \\
\text { (CFU/mL) }\end{array}$ \\
\hline $1.0 \times 10^{3}$ to $2.7 \times 10^{3}$ & $20: 00$ to $22: 40$ & $2.4 \times 10^{7}$ to $3.0 \times 10^{7}$ & \multirow{3}{*}{$2.5 \times 10^{8}$ to $3.2 \times 10^{8}$} & \multirow{3}{*}{$6.0 \times 10^{7}$ to $8.0 \times 10^{7}$} \\
\hline $1.0 \times 10^{2}$ to $2.3 \times 10^{2}$ & $23: 10$ to $30: 50$ & $1.6 \times 10^{7}$ to $5.6 \times 10^{7}$ & & \\
\hline $1.4 \times 10^{1}$ to $5.9 \times 10^{1}$ & $24: 40$ to $29: 10$ & $1.0 \times 10^{7}$ to $1.3 \times 10^{7}$ & & \\
\hline
\end{tabular}

\subsubsection{RAST Directly from Positive Blood Culture Vials}

Finally, we aimed to evaluate the applicability of EUCAST RAST directly from positive $Y$. pestis BC vials. We followed EUCAST guidelines and examined the possibility of performing RAST at three time points; when the BC bottles were flagged as positive, $18 \mathrm{~h}$ after they were flagged as positive and continued to incubate in the BACTEC ${ }^{\mathrm{TM}}$ FX40 device, and after they were flagged as positive and were kept at room temperature for an additional $3 \mathrm{~h}$. The positive BCs were tested using the same panel of potential therapeutic antibiotics as previously tested using standard colony-suspended bacterial cultures. For all antibiotics tested, inhibition zone diameters could be observed by RAST performed on the positive BCs as early as $18 \mathrm{~h}$ (Table 10 for ciprofloxacin and Supplementary Tables S15-S18 for tetracycline, gentamicin, chloramphenicol and trimethoprim-sulfamethoxazole).

Table 10. Ciprofloxacin inhibition zone diameters of $Y$. pestis-positive blood cultures.

\begin{tabular}{ccccc}
\hline & \multicolumn{3}{c}{ Inhibition Zone Diameter (mm) } \\
\hline Incubation Duration (h) & Standard Conditions ${ }^{\text {a }}$ & At the Alert & $\begin{array}{c}\text { 18 h after the Alert, } \\
\text { Incubation in the Device }\end{array}$ & $\begin{array}{c}\text { 3 h after the Alert, } \\
\text { at Room Temperature }\end{array}$ \\
\hline 18 & $35-40$ & $35-40$ & $37-39$ & $40-42$ \\
20 & $36-40$ & $36-40$ & $37-42$ & $38-42$ \\
22 & $39-41$ & $36-42$ & $37-42$ & 42 \\
24 & $39-42$ & 42 & $37-43$ & $43-45$ \\
\hline
\end{tabular}

${ }^{\text {a }}$ Ciprofloxacin inhibition zone diameter for the standard culture were taken from Table 7 ; the diameters were determined by at least three independent assays.

However, as there were differences in the inhibition zone diameters resulting directly from BCs compared to the diameters of standard conditions for some of the antibiotics, explicit breakpoint tables will be required for the implementation of RAST for Y. pestispositive BCs. In contrast to the disc-diffusion-based AST, using Etest strips for RAST (Table 11 for ciprofloxacin and Supplementary Tables S19-S23 for doxycycline, chloramphenicol, trimethoprim-sulfamethoxazole, gentamicin and streptomycin) resulted in MIC values that were similar to those obtained under standard conditions, starting from $18 \mathrm{~h}$ of incubation. These results support the usage of breakpoint interpretation tables that were determined for Y. pestis under standard conditions for Etest-based RAST at all time points, starting from an 18-hour incubation.

Table 11. Ciprofloxacin Etest MIC values of Y. pestis-positive blood cultures.

\begin{tabular}{cccc}
\hline & & MIC $(\mu \mathrm{g} / \mathrm{mL})$ \\
\hline Incubation Duration $(\mathbf{h})$ & Standard Culture ${ }^{\text {a }}$ & At the Alert & $\begin{array}{c}\mathbf{1 8} \text { h after the Alert, Incubation } \\
\text { in the Device }\end{array}$ \\
\hline 18 & 0.016 & $0.016-0.03$ & 0.016 \\
20 & 0.016 & $0.016-0.03$ & $0.016-0.03$ \\
22 & $0.016-0.03$ & $0.016-0.03$ & $0.016-0.03$ \\
24 & $0.016-0.03$ & 0.016 & $0.016-0.03$ \\
at Room Temperature & 0.016 \\
\hline
\end{tabular}

${ }^{a}$ Ciprofloxacin MIC values for the standard culture were taken from Table 8; the MIC values were determined by at least three independent assays. 


\subsection{Francisella tularensis}

\subsubsection{Early Determination of AST Results}

For F. tularensis, we first determined, for a panel of potential therapeutic antibiotics, the earliest time points for which reliable AST results can be obtained. For this purpose, we performed a disc-diffusion-based test using a standard F. tularensis suspension obtained from isolated colonies and determined the inhibition zone diameter at different time points. As F. tularensis does not grow on MHA, we used Muller Hinton Fastidious (MH-F) agar (purchased from two vendors), which is EUCAST's recommended growth medium for fastidious bacteria. Unfortunately, this growth medium also did not properly support the growth of $F$. tularensis, impeding its use for AST assays. We, therefore, performed assays using CHA, a solid growth medium commonly used for F. tularensis growth and antimicrobial susceptibility tests [39]. Distinct inhibition zone edges were evident as early as $24 \mathrm{~h}$ following incubation for all the antibiotics tested-CIP, TET, CHL and GEN (Table 12).

Table 12. Disc-diffusion inhibition zones of F. tularensis at various time points.

\begin{tabular}{ccccc}
\hline & \multicolumn{4}{c}{ Inhibition Zone Diameter (mm) } \\
\hline Incubation Duration (h) & CIP & TET & CHL & GEN \\
\hline 24 & $40-42$ & $39-40$ & $40-41$ & $23-25$ \\
$30-40$ & $42-44$ & $40-41$ & $41-43$ & $26-27$ \\
48 & $43-44$ & $40-42$ & $40-43$ & $27-28$ \\
\hline
\end{tabular}

CIP-ciprofloxacin; TET-tetracycline; CHL-chloramphenicol; GEN-gentamicin; the diameters were determined by at least three independent assays.

However, for CIP, TET and GEN, the inhibition zone diameters differed between $24 \mathrm{~h}$ and the standard reading times $(48 \mathrm{~h})$. Hence, to shorten the reading times to $24 \mathrm{~h}$, distinct breakpoint tables should be created. Reading the inhibition zone following a 30-hour and up incubation period, the inhibition zone diameters were similar to those observed at $48 \mathrm{~h}$ for all four tested antibiotics. When we used Etest strips to determine MIC values (Table 13), the MIC values at $24 \mathrm{~h}$ and at the standard reading time ( $48 \mathrm{~h}$ ) were similar; thus, using Etest-based RAST, proper MIC values could be read as early as $24 \mathrm{~h}$ of incubation, and breakpoint determination could be obtained from standard breakpoint tables, such as those published by CLSI [38].

Table 13. Etest MIC values of F. tularensis at various time points.

\begin{tabular}{cccccc}
\hline & \multicolumn{5}{c}{ MIC $(\mu \mathrm{g} / \mathrm{mL})$} \\
\hline Incubation Duration (h) & CIP & DOX & CHL & GEN & STR \\
\hline 24 & 0.016 & 0.25 & $0.5-1$ & $0.5-1$ & $2-4$ \\
$30-40$ & $0.008-0.016$ & $0.25-0.5$ & $0.5-1$ & $0.5-1$ & $2-4$ \\
48 & $0.008-0.016$ & $0.25-0.5$ & 0.5 & $0.5-1$ & $2-4$ \\
\hline
\end{tabular}

DOX-doxycycline; CIP—ciprofloxacin; CHL—chloramphenicol; GEN—gentamicin; STR—streptomycin; the MIC values were determined by at least three independent assays.

\subsubsection{Characterization of F. tularensis Growth in Human Blood Culture}

Next, we analyzed the growth rates of $F$. tularensis in $\mathrm{BC}$ vials and the bacterial concentrations when the vials were flagged as positive. We spiked F. tularensis into human blood with a wide range of bacterial concentrations (Table $14,2 \times 10^{1}$ to $2.9 \times 10^{6} \mathrm{CFU} / \mathrm{mL}$ ) and transferred the spiked blood into BACTEC ${ }^{\mathrm{TM}}$ Plus Aerobic/F culture vials, which were then incubated in the BACTEC ${ }^{\mathrm{TM}}$ FX40 device until the vials were marked as positive. The bacterial concentrations were counted at the alert and, following EUCAST guidelines, after an additional $18 \mathrm{~h}$ of incubation in the device or after an additional $3 \mathrm{~h}$ of incubation at room temperature. As observed for B. anthracis and Y. pestis, the F. tularensis concentrations at the device alert were at a narrow range of $\sim 1$ order of magnitude 
$\left(1.4 \times 10^{7}\right.$ to $3.6 \times 10^{8} \mathrm{CFU} / \mathrm{mL}$; "concentration at the alert" column) compared to the broad 5-orders-of-magnitude range in the inoculum concentrations.

Table 14. Characterization of F. tularensis growth in blood cultures.

\begin{tabular}{|c|c|c|c|c|}
\hline $\begin{array}{c}\text { Initial } \\
\text { Concentration in } \\
\text { Blood }(\mathrm{CFU} / \mathrm{mL})\end{array}$ & $\begin{array}{c}\text { Incubation Duration } \\
\text { until the Alert } \\
\text { (dd: hh: } \mathrm{mm} \text { ) }\end{array}$ & $\begin{array}{l}\text { Concentration at the } \\
\text { Alert (CFU/mL) }\end{array}$ & $\begin{array}{c}\text { Concentration, } 18 \mathrm{~h} \\
\text { after the Alert, Incubation } \\
\text { in the Device (CFU/mL) }\end{array}$ & $\begin{array}{c}\text { Concentration, } 3 \mathrm{~h} \text { after } \\
\text { the Alert, at Room } \\
\text { Temperature (CFU/mL) }\end{array}$ \\
\hline $2.6 \times 10^{6}$ to $2.9 \times 10^{6}$ & $0: 19: 00$ to $0: 23: 30$ & \multirow{3}{*}{$1.5 \times 10^{7}$ to $3.0 \times 10^{8}$} & \multirow{3}{*}{$3.6 \times 10^{8}$ to $2.3 \times 10^{9}$} & \multirow{3}{*}{ ND } \\
\hline $1.0 \times 10^{5}$ to $3.3 \times 10^{5}$ & 1:09:00 to $2: 06: 30$ & & & \\
\hline $2.6 \times 10^{4}$ to $2.8 \times 10^{4}$ & $2: 07: 30$ to $2: 14: 30$ & & & \\
\hline $2.6 \times 10^{3}$ to $4.9 \times 10^{3}$ & $3: 23: 00$ to $4: 17: 00$ & $1.4 \times 10^{7}$ to $9.0 \times 10^{7}$ & $1.4 \times 10^{8}$ to $8.0 \times 10^{8}$ & ND \\
\hline $2.0 \times 102$ to $4.8 \times 102$ & 4:22:00 to $8: 01: 30$ & \multirow{2}{*}{$1.6 \times 10^{7}$ to $3.6 \times 10^{8}$} & \multirow{2}{*}{$1.8 \times 10^{8}$ to $1.6 \times 10^{9}$} & \multirow{2}{*}{$5.0 \times 10^{7}$ to $5.6 \times 10^{8}$} \\
\hline $2.0 \times 101$ to $4.3 \times 101$ & $6: 13: 00$ to $12: 04: 00$ & & & \\
\hline
\end{tabular}

ND—not determined; the values were determined by at least three independent assays.

Moreover, the incubation duration until the vials were marked as positive was negatively correlated with inoculum concentrations, and while $\sim 1$ day was needed for $\sim 10^{6} \mathrm{CFU} / \mathrm{mL}$ spiked BC to become positive, 6 to 12 days were required for cultures spiked with 20 to $40 \mathrm{CFU} / \mathrm{mL}$ ("incubation duration until the alert" column). Further incubation of the $\mathrm{BC}$ s in the device or at room temperature (18 or $3 \mathrm{~h}$, respectively) showed that bacteria were still growing under those conditions. Hence, it is applicable to initiate RAST with postponed BCs. Notably, for BCs containing low concentrations of F. tularensis, the growth rates were very slow, exceeding the 5-day default incubation protocol used by the device to flag BCs as negative; thus, false-negative results could be obtained when low levels of F. tularensis were present in the blood. Furthermore, when we assessed F. tularensis growth in anaerobic $\mathrm{BC}$ vials, no growth was evident even when we grew the high concentrations of F. tularensis for 15 days.

\subsubsection{RAST Directly from Positive Blood Culture Vials}

Finally, we applied the EUCAST RAST directly from F. tularensis-positive BCs using the condition we found before to be optimal for F. tularensis RASTs, conducting both disc-diffusion- and Etest-based RASTs toward the four therapeutic antibiotics. We again determined the MIC values at the three EUCAST-recommended time points: when the BC bottles were flagged as positive, an additional $18 \mathrm{~h}$ incubation in the device and an additional $3 \mathrm{~h}$ incubation at room temperature after being positively flagged. Under these conditions, for each time point, the disc-diffusion inhibition zones were similar to those obtained with the standard F. tularensis inoculum (Table 15 for ciprofloxacin and Supplementary Tables S24-S26 for tetracycline, chloramphenicol and gentamicin).

Table 15. Ciprofloxacin inhibition zone diameters of F. tularensis-positive blood cultures.

\begin{tabular}{ccccc}
\hline & \multicolumn{3}{c}{ Inhibition Zone Diameter (mm) } \\
\hline Incubation Duration (h) & Standard Culture a & At the Alert & $\begin{array}{c}\text { 18 h after the Alert, } \\
\text { Incubation in the Device }\end{array}$ & $\begin{array}{c}\mathbf{3} \text { h after the Alert, } \\
\text { at Room Temperature }\end{array}$ \\
\hline 24 & $40-42$ & 40 & 40 & 40 \\
$30-40$ & $42-44$ & $43-44$ & $42-43$ & 43 \\
48 & $43-44$ & 44 & $43-44$ & 44 \\
\hline
\end{tabular}

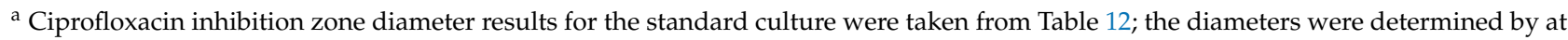
least three independent assays.

Again, distinct inhibition zones could be seen following $24 \mathrm{~h}$ of incubation, but at least a 30-hour incubation period was required to obtain results that were similar to those of the standard reading time $(48 \mathrm{~h})$. Using Etest strips, RAST MIC values similar to the standard 
MIC values could be obtained for all antibiotics as early as $24 \mathrm{~h}$ of incubation (Table 16 for ciprofloxacin and Supplementary Tables S27-S30 for doxycycline, chloramphenicol, gentamycin and streptomycin), emphasizing the advantage of using Etest strips to shorten the AST duration.

Table 16. Ciprofloxacin Etest MIC values of F. tularensis-positive blood cultures.

\begin{tabular}{ccccc}
\hline & & MIC $(\mu \mathrm{g} / \mathrm{mL})$ \\
\hline Incubation Duration (h) & Standard Culture ${ }^{\text {a }}$ & At the Alert & $\begin{array}{c}\text { 18 h after the alert, } \\
\text { Incubation in the Device }\end{array}$ & $\begin{array}{c}\text { 3 h after the Alert, } \\
\text { at Room Temperature }\end{array}$ \\
\hline 24 & 0.016 & $0.008-0.016$ & 0.016 & 0.016 \\
$30-40$ & $0.008-0.016$ & $0.008-0.016$ & $0.008-0.016$ & $0.008-0.016$ \\
48 & $0.008-0.016$ & $0.008-0.016$ & $0.008-0.016$ & $0.008-0.016$ \\
\hline
\end{tabular}

${ }^{\text {a }}$ Ciprofloxacin MIC values for the standard culture were taken from Table 13; the MIC values were determined by at least three independent assays.

\section{Discussion}

The initiation of effective antibiotic treatment is critical for treating the systemic spread of infectious bacterial pathogens. There is great importance for rapid and proper antibiotic treatment, which can increase survival rates and reduce hospitalization length and costs. Until the last few years, the standard method to perform AST assays from positive BCs required a quantified suspension of bacteria originating from agar-grown colonies. This method is time consuming and usually takes up to 2 days from the time that the $\mathrm{BC}$ vials were flagged positive by the automatic systems. Over the years, attempts to develop rapid AST assays for various human pathogens (for review, see [40]), including Tier 1 select agents [41-43], have been made. These methods can provide efficient, tailored and rapid treatment of bacterial infections. However, most of these assays are not standardized, restricting their worldwide acceptance. One of those approaches, which performs AST on a sample taken directly from a positive BC, was recently adopted by the EUCAST, the European committee responsible for promoting the standardization of AST methods used in Europe, encouraging its wide acceptance. This approach was validated for a limited number of common life-threatening bacteria that can easily acquire antibiotic resistance (Escherichia coli, Klebsiella pneumoniae, Pseudomonas aeruginosa, Acinetobacter baumannii, Staphylococcus aureus, Enterococcus faecalis, Enterococcus faecium and Streptococcus pneumoniae). However, there is a need to further extend the applicability of this method to additional pathogens.

In the current study, we examined the applicability of this direct approach to three Tier 1 select agents: $B$. anthracis, Y. pestis and F. tularensis. Due to the rapid and lifethreatening clinical implications of exposure to these bacteria, together with the potential of either natural emergence or malicious generation of resistant strains in bioterror scenarios, rapid AST assays for these bacteria are highly desirable. The proficiency of the EUCAST RAST was evaluated by determining the shortest times for which proper inhibition zone diameters were derived by disc-diffusion-based AST. Using disc-diffusion assays, the assay suggested by the EUCAST guidelines, we obtained reliable inhibition zone diameters as early as 6, 18 and $24 \mathrm{~h}$ for B. anthracis, Y. pestis and F. tularensis, respectively, for most of the tested therapeutic antibiotics. However, as the inhibition diameters varied slightly from those obtained at standard reading times (18 to 20, 24 and $48 \mathrm{~h}$ for B. anthracis, $Y$. pestis and F. tularensis, respectively), new breakpoint interpretation tables should be established for each bacterial-antibiotic combination, as was done by EUCAST for the validated bacteria. As the establishment of new breakpoint interpretation tables is a tedious task that requires validation with multiple strains, it is rarely performed even by standard committees such as the EUCAST. We, therefore, evaluated Etest-based AST as an alternative method to determine bacterial susceptibility. We found that Etest MIC values obtained at early time points were identical to those obtained at the standard reading times; thus, category breakpoint values could be based on standard interpretation tables, such as those 
already published by the CLSI [38], emphasizing the advantage of using the Etest assay over disc-diffusion AST. Another advantage of Etest-based RAST is the ability to determine MIC values in addition to the susceptibility category, which can be beneficial in some clinical cases, such as in pregnant women and children and in infections of intermediate strains or when the antibiotic susceptibility breakpoint concentration is not determined. On the downside, Etest strips are relatively expensive compared to discs and, therefore, are less suitable for usage in routine clinical laboratories that perform many AST assays each day. Nevertheless, since infection with these Tier 1 select agents is not common, the financial toll for using the Etest method for these bacteria is acceptable.

One of the factors that can affect AST results is the concentration of the bacterial inoculum used in the assay. For this reason, we characterized the growth of $B$. anthracis, $Y$. pestis and F. tularensis in a BACTEC ${ }^{\mathrm{TM}} \mathrm{FX} 40$ blood culture instrument, an automatic blood culturing system commonly used in clinical laboratories. This device monitors the accumulation of $\mathrm{CO}_{2}$ released by the growing bacteria using a fluorescent indicator present at the bottom of the $\mathrm{BC}$ vial and by using sophisticated algorithms to determine whether microbial growth is evident. The incubation time to positive BC alert and the bacterial concentrations at the device alert have not been previously reported for these bacteria. These data may be beneficial for the development of additional AST assays originating from grown BCs. We revealed that despite the marked difference in the bacterial concentrations that were initially spiked into the blood, when the vials were marked positive, the final concentrations were in a relatively limited range, $6.0 \times 10^{6}$ to $8.7 \times 10^{7} \mathrm{CFU} / \mathrm{mL}$, $1.0 \times 10^{7}$ to $5.6 \times 10^{7} \mathrm{CFU} / \mathrm{mL}$ and $1.4 \times 10^{7}$ to $3.6 \times 10^{8} \mathrm{CFU} / \mathrm{mL}$ for B. anthracis, Y. pestis and $F$. tularensis, respectively. The consistency in the final bacterial concentrations was accompanied by extension of the incubation duration for blood containing low levels of bacteria. This consistency may explain the omission of the bacterial quantification step of the positive BCs before plating for RAST, according to the EUCAST guidelines.

Importantly, we found that for low levels of F. tularensis-spiked blood, concentrations that are still clinically relevant, the standard 5-day incubation protocol, commonly used in clinical laboratories, is not sufficiently long and may result in false-negative BCs (Table 14). Notably, when we tried to grow F. tularensis in anaerobic BC vials, no growth was evident, even at the highest bacterial inoculum levels, indicating that these vials do not support $F$. tularensis growth. For comparison, $B$. anthracis growth in anaerobic vials was similar to its growth in aerobic vials, and $Y$. pestis grew faster (up to $6 \mathrm{~h}$ ) in the anaerobic vials; therefore, we used anaerobic vials to determine the RAST performance for $Y$. pestis.

Likewise, we found that the standard MH-F agar plates, which are recommended by the EUCAST guidelines for fastidious bacteria, did not support the growth of $F$. tularensis and, therefore, were not suitable for RAST or standard AST assays of $F$. tularensis. However, using CHA plates, a common medium used for growing and for MIC determination of F. tularensis [39], enabled the use of the RAST method to accurately determine the MIC values.

For all three tested bacteria, we could postpone the initiation of RAST, even after an additional $18 \mathrm{~h}$ of incubation in the device, without affecting the RAST results, suggesting that the assay can be conducted at convenient working hours in a clinical laboratory. Proper RAST results were also obtained after a delay of $3 \mathrm{~h}$ at room temperature of positive-flagged vials, allowing convenient transfer of the vials from one site to another.

Our results highlight the importance of validating the RAST assays with the relevant bacteria-antibiotic combinations before applying them to new bacteria. However, due to the lack of relevant strains (sensitive and resistant), we could not extensively validate the assay using more strains. This should be done by any laboratories wishing to implement this assay or by a central organization such as the EUCAST or the CLSI.

\section{Conclusions}

We have shown here that the EUCAST RAST method can be applied, with some modification, to the three Tier 1 select agents-B. anthracis, $Y$. pestis and F. tularensis- 
toward a panel of therapeutic antibiotics, when conducting the AST assays directly from positive BCs. The elimination of the preliminary enrichment step and shortening of the AST incubation time in the RAST assay compared to the standard AST assay can reduce labor, enable shorter time to result and most importantly lead to prompt adequate antibiotic treatment for these dangerous pathogens.

Supplementary Materials: The following are available online at https://www.mdpi.com/article/10 .3390 / microorganisms9051055/s1, Table S1: Tetracycline inhibition zone diameters of B. anthracispositive blood cultures. Table S2: Penicillin G inhibition zone diameters of B. anthracis-positive blood cultures. Table S3: Amoxicillin-clavulanic acid inhibition zone diameters of B. anthracis-positive blood cultures. Table S4: Linezolid inhibition zone diameters of B. anthracis-positive blood cultures. Table S5: Moxifloxacin inhibition zone diameters of B. anthracis-positive blood cultures. Table S6: Levofloxacin inhibition zone diameters of B. anthracis-positive blood cultures. Table S7: Imipenem inhibition zone diameters of $B$. anthracis-positive blood cultures. Table S8: Etest doxycycline MIC values for B. anthracis-positive blood cultures. Table S9: Etest ampicillin MIC values for B. anthracispositive blood cultures. Table S10: Etest clarithromycin MIC values for B. anthracis-positive blood cultures. Table S11: Etest chloramphenicol MIC values for B. anthracis-positive blood cultures. Table S12: Etest clindamycin MIC values for B. anthracis-positive blood cultures. Table S13. Etest vancomycin MIC values for B. anthracis-positive blood cultures. Table S14: Etest rifampicin MIC values for B. anthracis-positive blood cultures. Table S15: Tetracycline inhibition zone diameters of $Y$. pestis-positive blood cultures. Table S16: Gentamicin inhibition zone diameters of $Y$. pestis-positive blood cultures. Table S17: Chloramphenicol inhibition zone diameters of Y. pestis-positive blood cultures. Table S18: Trimethoprim-sulfamethoxazole inhibition zone diameters of Y. pestis-positive blood cultures. Table S19: Doxycycline Etest MIC values of Y. pestis-positive blood cultures. Table S20: Chloramphenicol Etest MIC values of Y. pestis-positive blood cultures. Table S21: Trimethoprimsulfamethoxazole Etest MIC values of $Y$. pestis-positive blood cultures. Table S22: Gentamicin Etest MIC values of Y. pestis-positive blood cultures. Table S23: Streptomycin Etest MIC values of $Y$. pestis-positive blood cultures. Table S24: Tetracycline inhibition zone diameters of $F$. tularensispositive blood cultures. Table S25: Chloramphenicol inhibition zone diameters of $F$. tularensis-positive blood cultures. Table S26: Gentamicin inhibition zone diameters of F. tularensis-positive blood cultures. Table S27: Doxycycline Etest MIC values of F. tularensis-positive blood cultures. Table S28: Chloramphenicol Etest MIC values of F. tularensis-positive blood cultures. Table S29: Gentamicin Etest MIC values of F. tularensis-positive blood cultures. Table S30: Streptomycin Etest MIC values of F. tularensis-positive blood cultures.

Author Contributions: Conceptualization, O.S., T.A., O.C., E.M., H.L., I.S.-L. and S.R.; methodology, O.S., T.A., M.A., D.G., H.C., E.B.-D., R.A.-G., I.S.-L. and S.R.; writing-original draft preparation, O.S., T.A., R.A.-G. and S.R.; writing-review and editing, O.S., T.A, M.A., D.G., H.C., O.C., E.M., H.L., R.A.-G., I.S.-L. and S.R.; supervision, O.S. and S.R. All authors have read and agreed to the published version of the manuscript.

Funding: This research received no external funding.

Institutional Review Board Statement: Not applicable.

Informed Consent Statement: Not applicable.

Data Availability Statement: Not applicable.

Conflicts of Interest: The authors declare no conflict of interest.

\section{References}

1. CDC. Bioterrorism Agents/Diseases. Available online: https://emergency.cdc.gov/agent/agentlist-category.asp (accessed on 12 May 2021).

2. Inglesby, T.V.; Dennis, D.T.; Henderson, D.A.; Bartlett, J.G.; Ascher, M.S.; Eitzen, E.; Fine, A.D.; Friedlander, A.M.; Hauer, J.; Koerner, J.F.; et al. Plague as a biological weapon: Medical and public health management. Working Group on Civilian Biodefense. JAMA 2000, 283, 2281-2290. [CrossRef] [PubMed]

3. Dennis, D.T.; Inglesby, T.V.; Henderson, D.A.; Bartlett, J.G.; Ascher, M.S.; Eitzen, E.; Fine, A.D.; Friedlander, A.M.; Hauer, J.; Layton, M.; et al. Tularemia as a biological weapon: Medical and public health management. JAMA 2001, 285, 2763-2773. [CrossRef] 
4. Inglesby, T.V.; O'Toole, T.; Henderson, D.A.; Bartlett, J.G.; Ascher, M.S.; Eitzen, E.; Friedlander, A.M.; Gerberding, J.; Hauer, J.; Hughes, J.; et al. Anthrax as a biological weapon, 2002: Updated recommendations for management. JAMA 2002, 287, $2236-2252$. [CrossRef]

5. Adalja, A.A.; Toner, E.; Inglesby, T.V. Clinical management of potential bioterrorism-related conditions. N Engl J. Med. 2015, 372, 954-962. [CrossRef] [PubMed]

6. Weiss, S.; Kobiler, D.; Levy, H.; Pass, A.; Ophir, Y.; Rothschild, N.; Tal, A.; Schlomovitz, J.; Altboum, Z. Antibiotics cure anthrax in animal models. Antimicrob. Agents Chemother. 2011, 55, 1533-1542. [CrossRef] [PubMed]

7. Sutera, V.; Levert, M.; Burmeister, W.P.; Schneider, D.; Maurin, M. Evolution toward high-level fluoroquinolone resistance in Francisella species. J. Antimicrob. Chemother. 2014, 69, 101-110. [CrossRef]

8. Galimand, M.; Carniel, E.; Courvalin, P. Resistance of Yersinia pestis to antimicrobial agents. Antimicrob. Agents Chemother. 2006, 50, 3233-3236. [CrossRef]

9. Pomerantsev, A.P.; Shishkova, N.A.; Marinin, L.I. Comparison of therapeutic effects of antibiotics of the tetracycline group in the treatment of anthrax caused by a strain inheriting tet-gene of plasmid pBC16. Antibiot. Khimioter. 1992, 37, 31-34.

10. Stepanov, A.V.; Marinin, L.I.; Pomerantsev, A.P.; Staritsin, N.A. Development of novel vaccines against anthrax in man. J. Biotechnol. 1996, 44, 155-160. [CrossRef]

11. Cabanel, N.; Bouchier, C.; Rajerison, M.; Carniel, E. Plasmid-mediated doxycycline resistance in a Yersinia pestis strain isolated from a rat. Int. J. Antimicrob. Agents 2018, 51, 249-254. [CrossRef]

12. Siebert, C.; Lindgren, H.; Ferre, S.; Villers, C.; Boisset, S.; Perard, J.; Sjostedt, A.; Maurin, M.; Brochier-Armanet, C.; Coute, Y.; et al. Francisella tularensis: FupA mutation contributes to fluoroquinolone resistance by increasing vesicle secretion and biofilm formation. Emerg. Microbes Infect. 2019, 8, 808-822. [CrossRef]

13. Karlsson, E.; Golovliov, I.; Larkeryd, A.; Granberg, M.; Larsson, E.; Ohrman, C.; Niemcewicz, M.; Birdsell, D.; Wagner, D.M.; Forsman, M.; et al. Clonality of erythromycin resistance in Francisella tularensis. J. Antimicrob. Chemother. 2016, 71, $2815-2823$. [CrossRef]

14. Sutera, V.; Hoarau, G.; Renesto, P.; Caspar, Y.; Maurin, M. In vitro and in vivo evaluation of fluoroquinolone resistance associated with DNA gyrase mutations in Francisella tularensis, including in tularaemia patients with treatment failure. Int. J. Antimicrob. Agents 2017, 50, 377-383. [CrossRef]

15. Fang, H.; Wang, H.; Cai, L.; Yu, Y. Prevalence of antibiotic resistance genes and bacterial pathogens in long-term manured greenhouse soils as revealed by metagenomic survey. Environ. Sci. Technol. 2015, 49, 1095-1104. [CrossRef]

16. Pilmis, B.; Thy, M.; Diep, J.; Krob, S.; Perillaud, C.; Couzigou, C.; Vidal, B.; Mizrahi, A.; Lourtet-Hascoet, J.; Le Monnier, A.; et al. Clinical impact of rapid susceptibility testing on MHR-SIR directly from blood cultures. J. Antimicrob. Chemother. 2019, 74, 3063-3068. [CrossRef]

17. Galar, A.; Yuste, J.R.; Espinosa, M.; Guillen-Grima, F.; Hernaez-Crespo, S.; Leiva, J. Clinical and economic impact of rapid reporting of bacterial identification and antimicrobial susceptibility results of the most frequently processed specimen types. Eur, J. Clin. Microbiol. Infect. Dis 2012, 31, 2445-2452. [CrossRef] [PubMed]

18. CLSI. Principles and Procedures for Blood Cultures, 1st ed.; Approved Guideline. CLSI standard M47-A; Clinical and Laboratories Standard Institute: Wayne, PA, USA, 2007.

19. Maugeri, G.; Lychko, I.; Sobral, R.; Roque, A.C.A. Identification and Antibiotic-Susceptibility Profiling of Infectious Bacterial Agents: A Review of Current and Future Trends. Biotechnol J. 2019, 14, e1700750. [CrossRef] [PubMed]

20. Idelevich, E.A.; Becker, K. How to accelerate antimicrobial susceptibility testing. Clin. Microbiol. Infect. 2019, 25, 1347-1355. [CrossRef]

21. Aloni-Grinstein, R.; Schuster, O.; Yitzhaki, S.; Aftalion, M.; Maoz, S.; Steinberger-Levy, I.; Ber, R. Isolation of Francisella tularensis and Yersinia pestis from Blood Cultures by Plasma Purification and Immunomagnetic Separation Accelerates Antibiotic Susceptibility Determination. Front. Microbiol. 2017, 8, 312. [CrossRef]

22. Steinberger-Levy, I.; Zahavy, E.; Cohen, S.; Flashner, Y.; Mamroud, E.; Aftalion, M.; Gur, D.; Ber, R. Enrichment of Yersinia pestis from blood cultures enables rapid antimicrobial susceptibility determination by flow cytometry. Adv. Exp. Med. Biol. 2007, 603, 339-350. [CrossRef]

23. Stokkou, S.; Geginat, G.; Schluter, D.; Tammer, I. Direct disk diffusion test using European Clinical Antimicrobial Susceptibility Testing breakpoints provides reliable results compared with the standard method. Eur. J. Microbiol. Immunol. $2015,5,103-111$. [CrossRef]

24. Jonasson, E.; Matuschek, E.; Kahlmeter, G. The EUCAST rapid disc diffusion method for antimicrobial susceptibility testing directly from positive blood culture bottles. J. Antimicrob. Chemother. 2020, 75, 968-978. [CrossRef]

25. Chandrasekaran, S.; Abbott, A.; Campeau, S.; Zimmer, B.L.; Weinstein, M.; Thrupp, L.; Hejna, J.; Walker, L.; Ammann, T.; Kirn, T.; et al. Direct-from-Blood-Culture Disk Diffusion To Determine Antimicrobial Susceptibility of Gram-Negative Bacteria: Preliminary Report from the Clinical and Laboratory Standards Institute Methods Development and Standardization Working Group. J. Clin. Microbiol. 2018, 56, e01678-17. [CrossRef] [PubMed]

26. EUCAST. Rapid AST directly from blood culture bottles. Available online: https://www.eucast.org/rapid_ast_in_blood_cultures/ (accessed on 12 May 2021).

27. Coyle, M.B.; McGonagle, L.A.; Plorde, J.J.; Clausen, C.R.; Schoenknecht, F.D. Rapid antimicrobial susceptibility testing of isolates from blood cultures by direct inoculation and early reading of disk diffusion tests. J. Clin. Microbiol. 1984, 20, 473-477. [CrossRef] 
28. Tan, T.Y.; Ng, L.S.; Kwang, L.L. Evaluation of disc susceptibility tests performed directly from positive blood cultures. J. Clin. Pathol. 2008, 61, 343-346. [CrossRef]

29. Doern, G.V.; Scott, D.R.; Rashad, A.L.; Kim, K.S. Evaluation of a direct blood culture disk diffusion antimicrobial susceptibility test. Antimicrob. Agents Chemother. 1981, 20, 696-698. [CrossRef]

30. Weme, E.T. Rapid antimicrobial susceptibility testing of positive blood cultures by direct inoculation and reading of disc diffusion tests after 3-4 hours. APMIS 2018, 126, 870-876. [CrossRef]

31. Perillaud, C.; Pilmis, B.; Diep, J.; Pean de Ponfilly, G.; Vidal, B.; Couzigou, C.; Mizrahi, A.; Lourtet-Hascoet, J.; Le Monnier, A.; Nguyen Van, J.C. Prospective evaluation of rapid antimicrobial susceptibility testing by disk diffusion on Mueller-Hinton rapid-SIR directly on blood cultures. Diagn. Microbiol. Infect. Dis. 2019, 93, 14-21. [CrossRef]

32. Rajshekar, D.; Chaudhari, K.V.; Bhat, P.; Prakash, S.S.; Raghvan, R.; Vasanth, S.; Jayakar, S.; Sugumaran, R.; Kannambath, R.; Chowdury, S.; et al. Evaluation of performance of direct disk diffusion test from positively flagged blood culture broth: A large scale study from South India. J. Lab. Physicians 2019, 11, 154-160. [CrossRef] [PubMed]

33. Froding, I.; Vondracek, M.; Giske, C.G. Rapid EUCAST disc diffusion testing of MDR Escherichia coli and Klebsiella pneumoniae: Inhibition zones for extended-spectrum cephalosporins can be reliably read after $6 \mathrm{~h}$ of incubation. J. Antimicrob. Chemother. 2017, 72, 1094-1102. [CrossRef] [PubMed]

34. Bianco, G.; Iannaccone, M.; Boattini, M.; Cavallo, R.; Costa, C. Assessment of rapid direct E-test on positive blood culture for same-day antimicrobial susceptibility. Braz J. Microbiol 2019, 50, 953-959. [CrossRef] [PubMed]

35. Levy, H.; Weiss, S.; Altboum, Z.; Schlomovitz, J.; Glinert, I.; Sittner, A.; Shafferman, A.; Kobiler, D. Differential contribution of Bacillus anthracis toxins to pathogenicity in two animal models. Infect. Immun 2012, 80, 2623-2631. [CrossRef]

36. Anonymous. Common buffers, media, and stock solutions. Curr. Protoc. Hum. Genet. 2001. [CrossRef]

37. Ben-Gurion, R.; Hertman, I. Bacteriocin-like material produced by Pasteurella pestis. J. Gen. Microbiol. 1958, 19, 289-297. [CrossRef] [PubMed]

38. CLSI. Methods for Antimicrobial Dilution and Disk Susceptibility Testing of Infrequently Isolated or Fastidious Bacteria, 3rd ed.; CLSI standard M45, CLSI standard M45 ed.; Clinical and Laboratories Standard Institute: Wayne, PA, USA, 2015.

39. Caspar, Y.; Maurin, M. Francisella tularensis Susceptibility to Antibiotics: A Comprehensive Review of the Data Obtained In Vitro and in Animal Models. Front. Cell Infect. Microbiol. 2017, 7, 122. [CrossRef]

40. Khan, Z.A.; Siddiqui, M.F.; Park, S. Current and Emerging Methods of Antibiotic Susceptibility Testing. Diagnostics 2019, 9, 49. [CrossRef] [PubMed]

41. Steinberger-Levy, I.; Shifman, O.; Zvi, A.; Ariel, N.; Beth-Din, A.; Israeli, O.; Gur, D.; Aftalion, M.; Maoz, S.; Ber, R. A Rapid Molecular Test for Determining Yersinia pestis Susceptibility to Ciprofloxacin by the Quantification of Differentially Expressed Marker Genes. Front. Microbiol. 2016, 7, 763. [CrossRef] [PubMed]

42. Shifman, O.; Steinberger-Levy, I.; Aloni-Grinstein, R.; Gur, D.; Aftalion, M.; Ron, I.; Mamroud, E.; Ber, R.; Rotem, S. A rapid antimicrobial susceptibility test for determining Yersinia pestis susceptibility to doxycycline by RT-PCR quantification of RNA markers. Front. Microbiol. 2019, 10, 754. [CrossRef]

43. Aloni-Grinstein, R.; Shifman, O.; Lazar, S.; Steinberger-Levy, I.; Maoz, S.; Ber, R. A rapid real-time quantitative PCR assay to determine the minimal inhibitory extracellular concentration of antibiotics against an intracellular Francisella tularensis Live Vaccine Strain. Front. Microbiol. 2015, 6, 1213. [CrossRef] 